\title{
Machine Learning Incorporating Host Factors for Predicting Survival in Head and Neck Squamous Cell Carcinoma Patients
}

\author{
Han Yu ${ }^{1}$, Sung Jun Ma ${ }^{2,3}{ }^{\circledR}$, Mark Farrugia ${ }^{2,3}{ }^{(D}$, Austin J. Iovoli ${ }^{2,3}$, Kimberly E. Wooten ${ }^{4}$, Vishal Gupta ${ }^{4}$, \\ Ryan P. McSpadden ${ }^{4}$, Moni A. Kuriakose ${ }^{4}$, Michael R. Markiewicz ${ }^{4,5,6}$, Jon M. Chan ${ }^{4}$, Wesley L. Hicks, Jr. ${ }^{4}$, \\ Mary E. Platek ${ }^{2,7,8}$ and Anurag K. Singh ${ }^{2, *}$
}

1 Department of Biostatistics and Bioinformatics, Roswell Park Comprehensive Cancer Center, Elm and Carlton Streets, Buffalo, NY 14263, USA; Han.Yu@roswellpark.org

2 Department of Radiation Medicine, Roswell Park Comprehensive Cancer Center, 665 Elm Street, Buffalo, NY 14203, USA; SungJun.Ma@RoswellPark.org (S.J.M.); Mark.Farrugia@roswellpark.org (M.F.); Austin.Iovoli@RoswellPark.org (A.J.I.); Mary.Platek@RoswellPark.org (M.E.P.)

3 Jacobs School of Medicine and Biomedical Sciences, University at Buffalo, The State University of New York, 955 Main Street, Buffalo, NY 14203, USA

4 Department of Head and Neck Surgery, Roswell Park Comprehensive Cancer Center, 665 Elm Street, Buffalo, NY 14203, USA; Kimberly.Wooten@RoswellPark.org (K.E.W.); Vishal.Gupta@RoswellPark.org (V.G.); Ryan.McSpadden@RoswellPark.org (R.P.M.); Moni.Kuriakose@RoswellPark.org (M.A.K.); Michael.Markiewicz@RoswellPark.org (M.R.M.); Jon.Chan@RoswellPark.org (J.M.C.); Wesley.Hicks@RoswellPark.org (W.L.H.J.)

check for

updates

Citation: Yu, H.; Ma, S.J.; Farrugia, M.; Iovoli, A.J.; Wooten, K.E.; Gupta, V.; McSpadden, R.P.; Kuriakose, M.A.; Markiewicz, M.R.; Chan, J.M.; et al. Machine Learning Incorporating Host Factors for Predicting Survival in Head and Neck Squamous Cell Carcinoma Patients. Cancers 2021, 13, 4559. https://doi.org/10.3390/ cancers13184559

Academic Editors: Alan Hutson and Song Liu

Received: 11 August 2021

Accepted: 7 September 2021

Published: 11 September 2021

Publisher's Note: MDPI stays neutral with regard to jurisdictional claims in published maps and institutional affiliations.

Copyright: (C) 2021 by the authors. Licensee MDPI, Basel, Switzerland. This article is an open access article distributed under the terms and conditions of the Creative Commons Attribution (CC BY) license (https:/ / creativecommons.org/licenses/by/ $4.0 /)$.
5 Department of Oral and Maxillofacial Surgery, School of Dental Medicine, University at Buffalo, The State University of New York, 3435 Main Street, Buffalo, NY 14214, USA

6 Department of Neurosurgery, Department of Surgery, Jacobs School of Medicine and Biomedical Sciences, University at Buffalo, The State University of New York, 955 Main Street, Buffalo, NY 14203, USA

7 Department of Cancer Prevention and Control, Roswell Park Comprehensive Cancer Center, 665 Elm Street, Buffalo, NY 14203, USA

8 Department of Dietetics, D'Youville College, 270 Porter Avenue, Buffalo, NY 14201, USA

* Correspondence: Anurag.Singh@RoswellPark.org; Tel.: +1-716-845-5715; Fax: +1-716-845-7616

Simple Summary: Among head and neck squamous cell carcinoma patients, the five-year survival rates have seen little improvement over the past decade. Prediction of a cancer patient's clinical outcome is challenging but important for patient counseling and treatment planning. In this work, we evaluated common machine learning models in predicting head and neck squamous cell carcinoma patients' overall survival based on clinical, demographic features and host factors. We identified the top-performing model and verified host factors can improve the model performance when proper methods are applied. The findings are of critical importance for improved risk stratification of head and neck squamous cell carcinoma patients and provide targeted supportive care for patients who are likely to have the worst outcome.

Abstract: Prognostication for cancer patients is integral for patient counseling and treatment planning, yet providing accurate prediction can be challenging using existing patient-specific clinical indicators and host factors. In this work, we evaluated common machine learning models in predicting head and neck squamous cell carcinoma (HNSCC) patients' overall survival based on demographic, clinical features and host factors. We found random survival forest had best performance among the models evaluated, which achieved a C-index of 0.729 and AUROC of 0.792 in predicting two-year overall survival. In addition, we verified that host factors are independently predictive of HNSCC overall survival, which improved the C-index by a margin of 0.026 and the AUROC by 0.034 . Due to the strong correlation among host factors, we showed that proper dimension reduction is an important step before their incorporation into the machine learning models, which provides a host factor score reflecting the patients' nutrition and inflammation status. The score by itself showed excellent discriminating capacity with the high-risk group having a hazard ratio of 3.76 (1.93-7.32, $p<0.0001)$ over the low-risk group. The hazard ratios were further improved to 7.41 (3.66-14.98, $p<0.0001$ ) by the random survival forest model after including demographic and clinical features. 
Keywords: overall survival; random survival forest; stratification; head and neck neoplasms

\section{Introduction}

Among patients with head and neck squamous cell carcinoma (HNSCC), 5-year survival rates have seen little improvement over the past decade, and, except for HPVassociated oropharyngeal cancers, remain below $50 \%$ for locally advanced disease [1]. The current treatment approach is to treat advanced cancers with multimodal therapies. This approach, however, carries significant complication rates and comorbidities. Studies supporting aggressive adjuvant chemotherapy and RT regimens, for example, argue for the need to recognize those patients that will fail traditional treatment regimens and offer them new treatment paradigms [2-4]. Therefore, it would be of clinical utility to discriminate between those patients who may or may not respond well to multimodality therapies, or to identify patients who would benefit from less toxic intervention.

In addition to well-known factors such as performance status and disease stage, prior work has demonstrated that patient-specific variables and host factors also influence $\mathrm{HN}$ SCC patient survival. The host factors can reflect the patient's immune, inflammation and nutritional status. It is therefore also logical to assume the host factors are associated with the host-tumor interaction in an interdependent manner. For example, decreased hemoglobin is a significant contributor to a hypoxic tumor environment [5], and impaired oxygen distribution through anemia can contribute to tumor hypoxia and consequently radio resistance [6-9]. Furthermore, elevated neutrophils can promote an inflammatory tumor microenvironment that can facilitate several oncologic processes, including suppression of the antitumor immune response [10-13]. Recent evidence suggests that pretreatment values of neutrophils, monocytes, lymphocytes, hemoglobin and albumin, are independently associated with prognosis in patients with HNSCC [5]. While the findings were important, these factors need to be validated using multivariate methods that consider multiple clinical factors in practicable decision models.

Machine learning tools such as random forests [14] (RF) often show superior performance over linear models in classification and regression tasks. The random survival forest (RSF) has extended application of RF model to censored time-to-event data [15], which is well suited to handle multiple, interrelated factors and potential modifiers. In this study, we used and compared both linear and non-linear machine learning tools to optimize the combination of known and novel predictors and thus maximize the prediction accuracy for patients' overall survival.

\section{Materials and Methods}

\subsection{Study Cohort}

Using a retrospective single-institution database, we reviewed and identified 591 primary HNSCC patients treated at Roswell Park Comprehensive Cancer Center with definitive or post-operative RT between 2003 and 2017. All patients selected for analysis have completed RT. Institutional review board approval was obtained (EDR 103707).

\subsection{Machine Learning Models}

The RSFs are generalizations of RFs for analyzing time-to-event data, which are tree-based ensemble machine learning techniques. Comparing with linear models, the RF model typically shows higher prediction performance because it naturally handles nonlinear relationships and complex interactions among predictors. For a new patient, the RSF predicts the survival probability at any time point (survival function) within eight years after treatment and the cumulative hazard function. The variable importance was used to quantify the contribution of each independent variable to the model predictions. In addition, we evaluated the performance of the Cox proportional hazards model with Least Absolute Shrinkage and Selection Operator (LASSO) regularization (COX). We further 
compared the DeepSurv, a recently developed Cox proportional hazards deep learning model [16]. Moreover, we also compared the performance of common machine learning methods for discriminating patient survival at given time points (year 1 5 after treatment). The methods evaluated include logistic regression with LASSO regularization [17], RF for classifications [14], extreme gradient boosting machine (GBM) [18] and artificial neural networks (ANN) [19]. For each time point, we built a separate model using each of these methods.

\subsection{Performance Metrics}

The model prediction performance in terms of overall survival (OS) was evaluated by concordance index (C-index). The $\mathrm{C}$-index is a generalization of area under the curves (AUCs) that accounts for the censored data. It is defined as the proportion of concordant pairs out of the total number of evaluable pairs. A pair is concordant if the subject with higher predicted probability of survival also has longer survival time. We also evaluated the model's performance in predicting patients' survival at year $1 \sim 5$. Specifically, a binary indicator of whether a patient's survival time is greater than a given time point was used as the outcome. Patients censored before the time point were excluded from this assessment. The receiver operating characteristic (ROC) curves were obtained by comparing the predicted risk against the binary outcome. An optimal cutoff was selected by maximizing the Youden index (sensitivity + specificity -1 ) of the ROC at year 2 . The sensitivity, specificity, positive predictive value (PPV) and negative predictive value (NPV) were reported. Calibration of the selected model was examined by comparing the observed and predicted probability at year $1 \sim 5$.

\subsection{Modeling Strategy}

Before any steps of model training, the cohort was randomly split into a training/validation set with $70 \%$ of subjects and a test set with $30 \%$ of subjects. For hosting factors, the observations with levels outside the range of mean $\pm 3 \mathrm{SD}$ (standard deviations) were trimmed at corresponding limits. Independent variables were standardized to have zero mean and unit variance before model training within each set separately. Missing values were imputed using $k$-nearest neighbors $(k N N)$ with $k=10$. Imputation was performed without involving the outcome variables and strictly within the training, validation and test cohorts. A standard principal component analysis (PCA) was used for dimension reduction in the standardized host factors. The PCA was performed only within the training/validation set. The PC scores of the test cohort were predicted by multiplying the training set loading matrix with the standardized test host factor levels. The standardization was performed based on the means and standard deviations of the training set to simulate the real-world prediction settings. We emphasize that no test data set was used in any model training steps, including pre-processing or unsupervised learning by PCA. The tuning of hyperparameters and model selection was performed using cross-validations (CV). The details can be found in the Supplementary Materials File S1.

\subsection{Model Interpretation}

For RSF, the variable importance (VIMP) will be used to quantify the contribution of each independent variable to the model predictions. VIMP measures the decrease in prediction performance (C-index) for the forest ensemble when a variable is randomly permuted. A large positive VIMP shows that the prediction accuracy of the forest is substantially degraded when this variable is noised-up by permutations. Therefore, a large VIMP indicated a potentially predictive variable. Partial dependence plots (PDPs) were used to show the marginal effect of a predictor on the predicted outcome [15]. The details can be found in the Supplementary Materials. 


\subsection{Statistical Analysis}

Spearman's correlation coefficient was used for the correlative analysis among host factors. Hierarchical clustering was performed based on Euclidean distance and complete linkage. The standard errors of C-index and AUROC were estimated using bootstrap methods with 1000 re-samplings. For cross-validation, the standard errors were estimated by the standard deviations of C-indices across runs. The results are presented by mean (SE) unless otherwise specified. For risk stratification, the survival curves were estimated using Kaplan-Meier product limit estimators. The hazard ratios (HRs) were estimated based on Cox proportional hazards models and the $95 \%$ confidence intervals (CIs) were reported. The analyses were conducted using R 4.1.0 and R packages randomForestSRC [20], glmnet [21], xgboost [18], neuralnet [19]. DeepSurv was implemented using PySurvival package [22] under Python 3.7.

\section{Results}

\subsection{Patient Characteristics}

The patient characteristics are summarized in Table 1. Among the study cohort, $62 \%$ of the patients received CCRT while $18 \%$ received CCRT and surgical treatment. The demographic and clinical features of patients are listed in Tables 1 and 2. The body mass index (BMI) and Karnofsky performance status were measured prior to the starting of radiation. Ethnicity and diagnosis type were not used as candidate predictors as the study cohort is highly homogeneous regarding these two characteristics. A summary of the host factors measured is shown in Table 2 . The event rate is $40 \%$ within eight years after treatment. The median follow-up of the cohort is 3.27 years, defined as the observation time for those event-free. The cohort was randomly split into a training/validation set $(70 \%, n=414)$ and test set $(30 \%, n=177)$.

Table 1. Summary of patient characteristics.

\begin{tabular}{|c|c|c|c|c|}
\hline Variable & Value & Frequency/Median & Percentage/IQR & Total \\
\hline Age & & 60.49 & $(54.22,66.86)$ & 591 \\
\hline BMI & & 27.09 & $(23.82,30.24)$ & 573 \\
\hline Weight loss & & 6 & $(2.5,9.8)$ & 565 \\
\hline $\begin{array}{c}\text { Karnofsky Performance } \\
\text { Status }\end{array}$ & & 9 & $(8,10)$ & 591 \\
\hline $\begin{array}{l}\text { Dose of primary } \\
\text { radiotherapy }\end{array}$ & & 70 & $(70,70)$ & 587 \\
\hline Radiotherapy duration & & 46 & $(45,46)$ & 591 \\
\hline \multirow{2}{*}{ Gender } & Male & 483 & $81.7 \%$ & \multirow[t]{2}{*}{591} \\
\hline & Female & 108 & $18.3 \%$ & \\
\hline \multirow{2}{*}{ Marital status } & Married & 298 & $50.4 \%$ & \multirow[t]{2}{*}{591} \\
\hline & Other & 293 & $49.6 \%$ & \\
\hline \multirow{2}{*}{ Anti-coagulants } & No & 546 & $92.4 \%$ & \multirow[t]{2}{*}{591} \\
\hline & Yes & 45 & $7.6 \%$ & \\
\hline \multirow{2}{*}{ NSAIDs } & $\mathrm{No}$ & 322 & $54.5 \%$ & \multirow[t]{2}{*}{591} \\
\hline & Yes & 269 & $45.5 \%$ & \\
\hline \multirow{3}{*}{ Alcohol consumption } & Never & 105 & $18.6 \%$ & \multirow[t]{3}{*}{566} \\
\hline & Former & 124 & $21.9 \%$ & \\
\hline & Current & 337 & $59.5 \%$ & \\
\hline \multirow{3}{*}{ Smoking status } & Never & 131 & $22.2 \%$ & \multirow[t]{3}{*}{591} \\
\hline & Former & 301 & $50.9 \%$ & \\
\hline & Current & 159 & $26.9 \%$ & \\
\hline
\end{tabular}


Table 1. Cont.

\begin{tabular}{|c|c|c|c|c|}
\hline Variable & Value & Frequency/Median & Percentage/IQR & Total \\
\hline \multirow{8}{*}{ Site } & Oral cavity/lip & 67 & $11.3 \%$ & 591 \\
\hline & Oropharynx & 257 & $43.5 \%$ & \\
\hline & Hypopharynx & 43 & $7.3 \%$ & \\
\hline & Nasopharynx & 15 & $2.5 \%$ & \\
\hline & Larynx & 142 & $24.0 \%$ & \\
\hline & Salivary gland & 9 & $1.5 \%$ & \\
\hline & Not specified & 52 & $8.8 \%$ & \\
\hline & Other & 6 & $1 \%$ & \\
\hline \multirow{4}{*}{ Clinical stage } & I & 18 & $3.1 \%$ & 575 \\
\hline & II & 46 & $8 \%$ & \\
\hline & III & 454 & $79 \%$ & \\
\hline & IV & 57 & $9.9 \%$ & \\
\hline \multirow{4}{*}{ Pathological grading } & Well differentiated & 40 & $8.4 \%$ & \\
\hline & Moderately differentiated & 227 & $47.4 \%$ & \\
\hline & Poorly differentiated & 204 & $42.6 \%$ & \\
\hline & Undifferentiated & 8 & $1.7 \%$ & \\
\hline \multirow{2}{*}{$\mathrm{HPV}$} & Negative & 131 & $39.3 \%$ & 333 \\
\hline & Positive & 202 & $60.7 \%$ & \\
\hline \multirow{6}{*}{ Treatment type } & RT only & 33 & $5.6 \%$ & 591 \\
\hline & CCRT & 364 & $61.6 \%$ & \\
\hline & Surgery + CCRT & 106 & $17.9 \%$ & \\
\hline & Surgery + RT & 25 & $4.2 \%$ & \\
\hline & CCRT + Neck Dissection & 7 & $1.2 \%$ & \\
\hline & $\mathrm{ICT}+\mathrm{CCRT}$ & 56 & $9.5 \%$ & \\
\hline \multirow{2}{*}{ Primary chemotherapy type } & Other or no chemotherapy & 136 & $23 \%$ & 591 \\
\hline & Cisplatin & 455 & $77 \%$ & \\
\hline \multirow{2}{*}{ Radiotherapy delayed } & No & 565 & $96.6 \%$ & 585 \\
\hline & Yes & 20 & $3.4 \%$ & \\
\hline \multirow{2}{*}{ Type of radiation } & Definitive & 474 & $80.2 \%$ & 591 \\
\hline & Post-operative (adjuvant) & 117 & $19.8 \%$ & \\
\hline \multirow{2}{*}{ Laterality of radiation } & Unilateral & 99 & $32.6 \%$ & 304 \\
\hline & Bilateral & 205 & $67.4 \%$ & \\
\hline \multirow{2}{*}{ Feeding tube type } & No & 250 & $42.3 \%$ & 591 \\
\hline & Yes & 341 & $57.7 \%$ & \\
\hline \multirow{2}{*}{ Hospitalized } & No & 463 & $78.5 \%$ & 590 \\
\hline & Yes & 127 & $21.5 \%$ & \\
\hline
\end{tabular}

Table 2. Summary of host factors.

\begin{tabular}{cccc}
\hline Variable & $\boldsymbol{N}$ & Median & IQR \\
\hline WBC & 591 & 7.25 & $(6.05,9.13)$ \\
HGB & 591 & 13.5 & $(12.1,14.75)$ \\
HCT & 591 & 40.1 & $(36.4,43.3)$ \\
RBC & 591 & 4.44 & $(3.96,4.81)$ \\
MCV & 591 & 90.7 & $(87.1,93.9)$ \\
MCH & 590 & 30.7 & $(29.5,31.9)$ \\
MCHC & 591 & 33.8 & $(33,34.4)$ \\
Neutrophil (\%) & 591 & 65.8 & $(59.15,72.4)$ \\
Lymphocyte (\%) & 591 & 23.9 & $(18.6,30)$ \\
Monocyte (\%) & 591 & 6.2 & $(1.5 .7 .5)$ \\
Eosinophil (\%) & 591 & 2.5 & $(0.4,0.7)$ \\
Basophil (\%) & 590 & 0.5 & \\
\hline
\end{tabular}




\subsection{Host Factors Organized as Clusters}

Correlative analyses within the training/validation cohort revealed two major clusters of the host factors. Figure 1A shows the absolute values of the pairwise Spearman's correlation coefficients among the host factors. Highly correlated factors tend to cluster together and reflect the status of an underlying biological process. A hierarchical clustering shows one mutually correlated group of $\mathrm{MCHC}, \mathrm{MCV}$ and $\mathrm{MCH}$. A second cluster mainly corresponds to the nutrition status or ability in oxygen delivering. The third cluster primarily reflects the immune or inflammatory status. A closer examination of the third cluster shows the neutrophil and WBC percentages form a strongly correlated group, which is negatively correlated with lymphocyte percentage (Figure 1B). Considering the strong correlations, we performed PCA on the HF variables. Consistent with Figure 1A,B, the biplot (Figure 1C) also shows three groups of variables. The biplot shows the projections (contributions) of each host factor to the first two principal components. A large horizontal (vertical) projection suggests the host factor primarily contributes to the PC1 (PC2). Generally, correlated factors tend to have projections to the same or opposite directions (positively/negatively correlated). The groups with projections in the bottom-left and upper-right quadrants correspond well to the lower cluster in Figure 1A, which reflects inflammatory status. Note that the factors in this cluster also forms two negatively correlated groups, as shown in Figure 1B. The other group in upper-left quadrant corresponds to the bottom cluster in Figure 1A. Although these two groups are mutually independent (projections are perpendicular), the first principal component (PC1) constitutes a composite score of these two domains in opposite directions. We define a host factor (HF) score as HF score $=$ PC1, then a higher HF score corresponds to a higher inflammation and poorer nutrition status.

\subsection{Model Selection and Evaluation}

The COX and RSF models' performance were compared using the CV within the training/validation set. Adding large number of strongly correlated features is generally harmful to a model's performance. Therefore, we postulate that including the HF features naively will not provide optimal model performance. Instead, the scores derived from PCA will be naturally uncorrelated summarizing features. We sequentially added the PCs ranked by their eigen values into the model and compared their performance (Table 3). Indeed, adding all HF features naively reduced the performance of RSF and did not maximize COX models' performance (RSF-ALL/COX-ALL) comparing with the models with clinical/demographic features alone (RSFc/COXc). Using the elastic net or ridge penalties which are better suited for correlated features only showed negligible difference. On the other hand, inclusion of PC scores improved both models' performance. Overall, the RSF model with only PC1 (HF score) showed best performance. Inclusion of more components does not further improve the validation performance. The COX-1, COX-2 and COX-3 models are identical because PC2 and PC 3 were not selected by the model, which further suggests that only PC1 (HF score) is a useful predictor. The superiority of RSF over COX model suggests a nonlinear relationship between predictors and the patient survival or complex interaction among the predictors, which cannot be captured by linear models. 
A

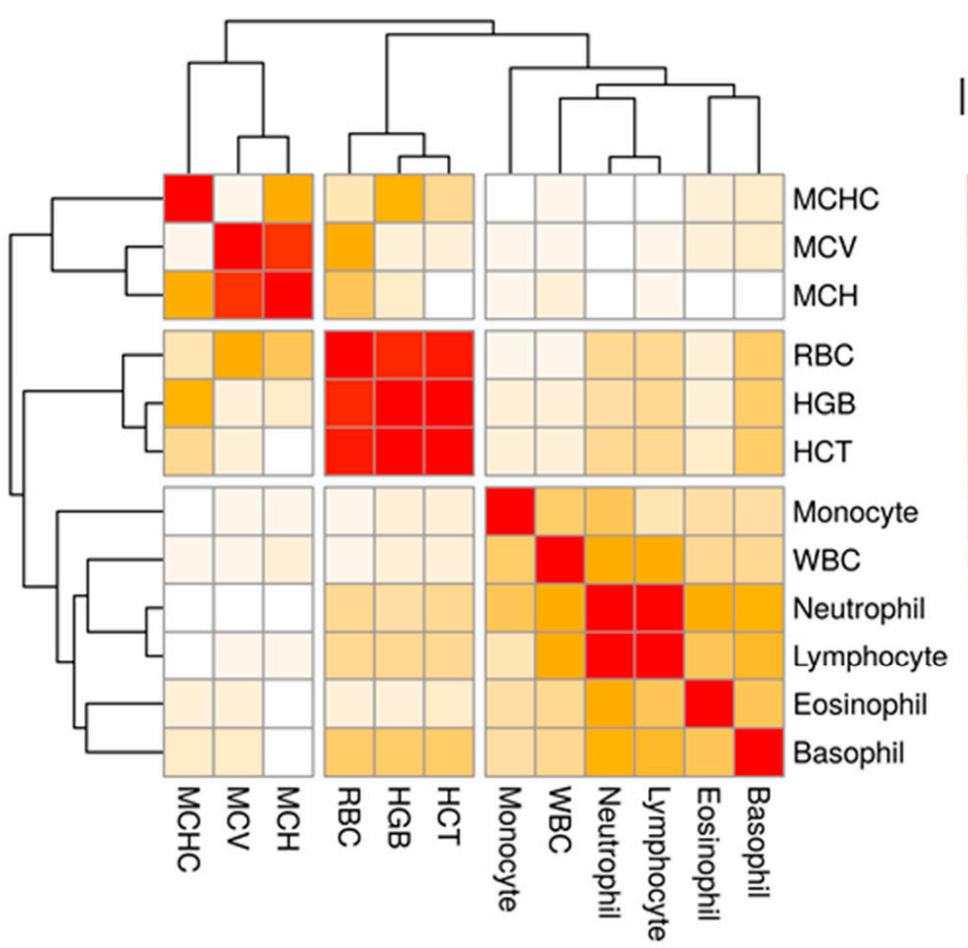

B

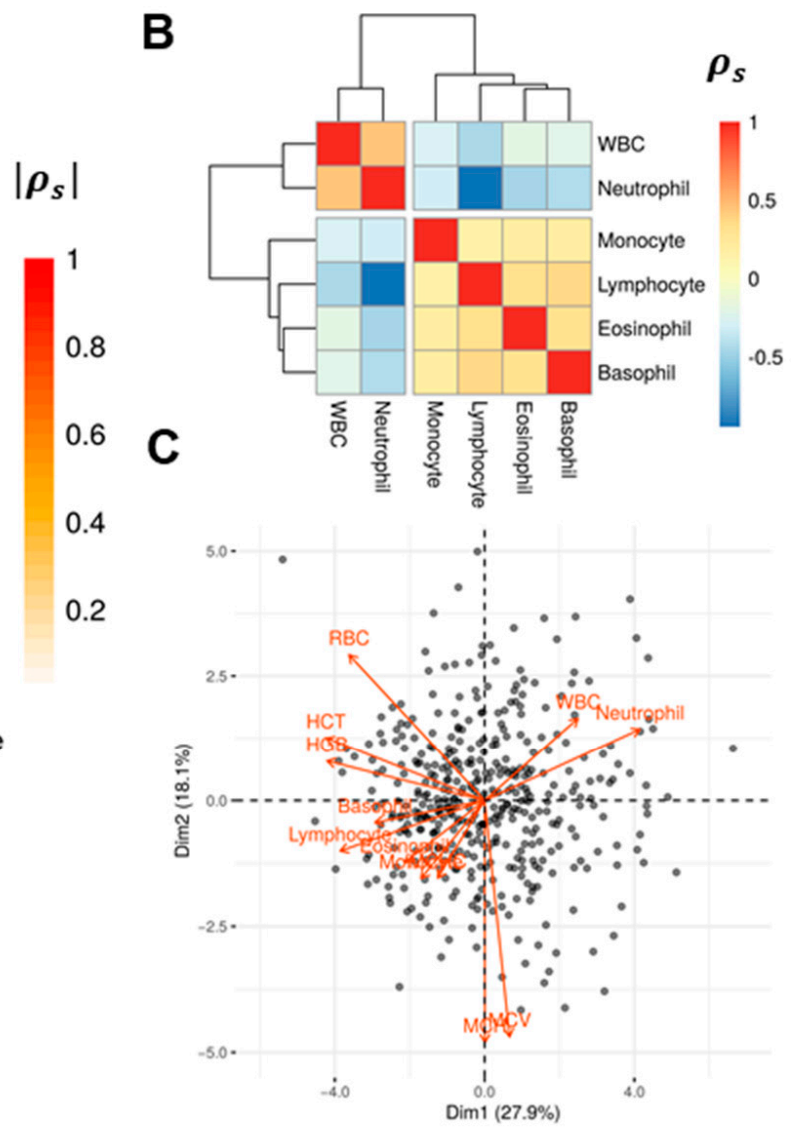

Figure 1. The host factors are mutually correlated. (A) The heatmap of the absolute Spearman correlation $\left(\left|\rho_{S}\right|\right)$ between host factors and the hierarchical clustering based on correlations. The correlations were obtained using the training cohort data. (B) The heatmap of the Spearman correlation $\left(\rho_{s}\right)$ between host factors in the first cluster. (C) The biplot of the PCA of the host factors. The biplot shows the projections (contributions) of each host factor to the first two principal components. A large horizontal (vertical) projection suggests the host factor primarily contributes to the PC1 (PC2). Two host factors with projections to the same (opposite) direction suggest they are positively (negatively) correlated with projections onto other PCs ignored. Perpendicular projections imply independence, for example RBC and WBC. The variance explained by each component are shown in corresponding axis labels.

Table 3. Model performance evaluated by the average C-index from cross-validations.

\begin{tabular}{cc}
\hline Model & Validation C-Index \\
\hline RSFc & $0.707(0.032)$ \\
RSF-1 & $0.721(0.013)$ \\
RSF-2 & $0.717(0.013)$ \\
RSF-3 & $0.717(0.009)$ \\
RSF-ALL & $0.705(0.015)$ \\
COXc & $0.671(0.042)$ \\
COX-1 & $0.690(0.024)$ \\
COX-2 & $0.690(0.024)$ \\
COX-3 & $0.690(0.024)$ \\
COX-ALL & $0.686(0.021)$ \\
\hline
\end{tabular}

In the test set, we further compared RSF's performance with COX model and the RSF model with only clinical/demographic features. Consistent with the CV results, the RSF model performed best in terms of C-index and AUCs (Figure 2A, Table 4). It also showed better performance than DeepSurv. This is not surprising since deep neural network models are often advantageous when sufficient amount of training data available. DeepSurv model is typical successfully applied when there are over thousands of records available for model 
training [16,23-25]. The C-index we obtained using DeepSurv is also close to the result in [23]. Another common strategy for predicting clinical outcome is to treat the prediction of survival at given times points as classification problems. Therefore, performance of the models trained above was further compared with common classification machine learning algorithms in predicting the survival at year 1 5. The methods tested include logistic regression with LASSO regularization (logistic), RF, GBM and ANN. Note that because we are treating the prediction of survival at each time point as separate classification problems, an independent model was trained for each time point in the training/validation set. This is different from RSF, which can predict the survival probability for any time point based on a single model. The result shows that RSF outperforms all classification models in terms of AUC (Figure 2B, Table 4). Only the RF and GBM models showed a better performance at year 1 . This is probably because the classification methods cannot fully utilize the timeto-event information. Model calibration of RSF was examined by comparing the predicted and actual two-year survival probability (Figure 2C).
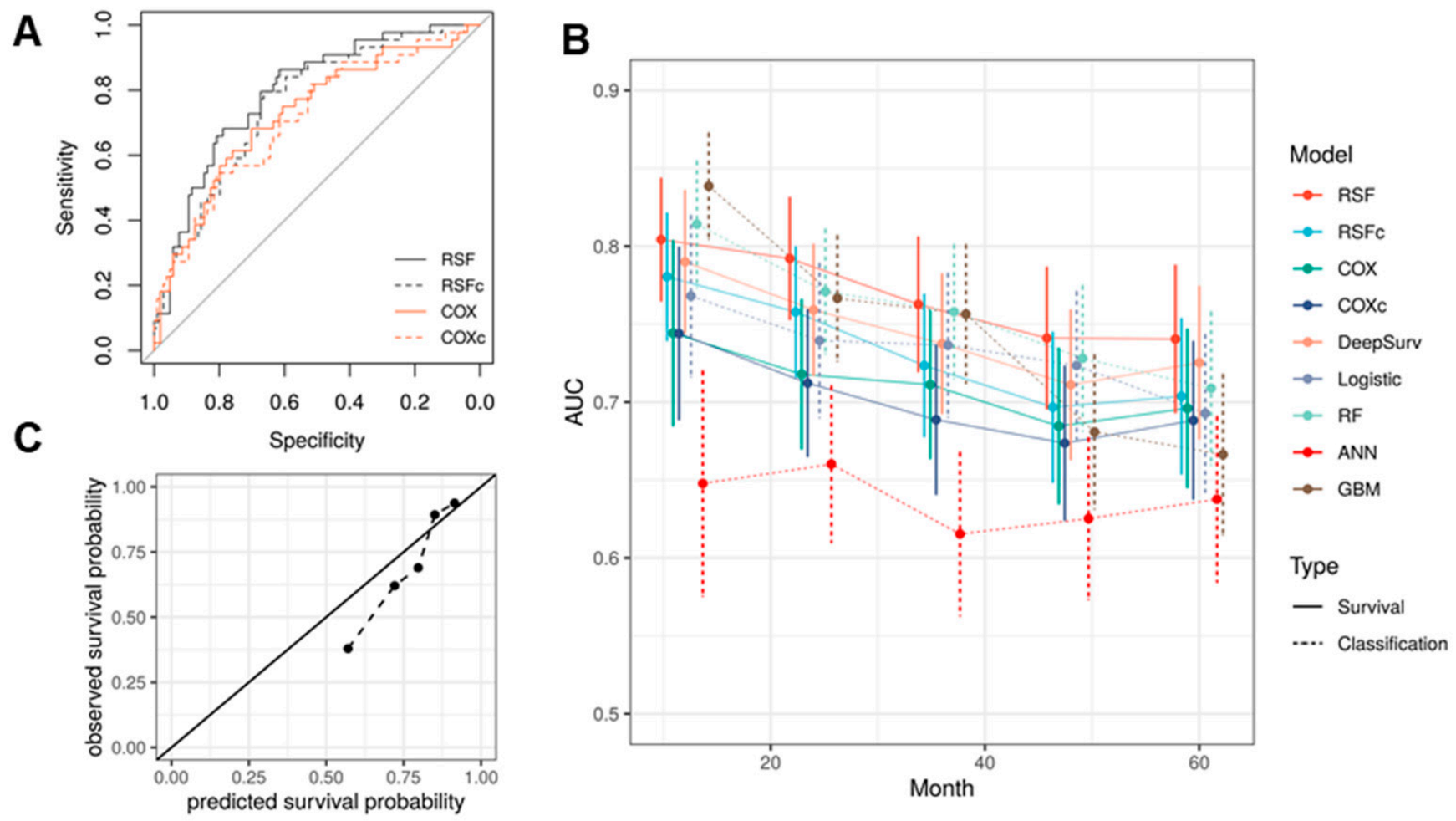

Figure 2. RSF predicts HNSCC patients' overall survival. (A) The ROC curves of the predicted risk by the survival models against observed survival at year two. (B) RSF achieved highest AUCs in predicting OS at year 2 5 among the models tested. (C) Model calibration of RSF by comparing the predicted and observed two-year survival probability. The black solid line denotes the perfect calibration line.

Variable importance plot shows that the HF score is ranked as one of the top three variables contributing to the prediction (Figure $3 \mathrm{~A}$ ). As a baseline, the variable alone can achieve a C-index of 0.656 (0.034), while the other two top-ranked variables BMI and KPS alone achieve C-indices of 0.648 (0.034) and $0.598(0.033)$, respectively.

As shown above, the better performance of RSF over COX model suggest non-linear relationships. This is supported by the partial dependence plots (Figure 3B), which show the relationship between the certain predictors and patients' two-year survival is not linear or even monotonic. For example, the two-year survival rate increases as BMI increases up to 25 , but then plateaus, and even decreases as the BMI further increases. The partial dependence plot shows an overall negative relationship between HF score and two-year 
survival (Figure 3C), i.e., patients with poorer nutrition and higher inflammation status tend to have worse OS, which is consistent with the previous findings.

Table 4. Model test performance measured by C-index and AUC of two-year survival. The cutoffs were selected by maximizing the Youden index. For classification models, the C-index is obtained based on the predicted two-year survival probability.

\begin{tabular}{|c|c|c|c|c|c|c|}
\hline Model & C-Index & AUC & Specificity & Sensitivity & PPV & NPV \\
\hline RSF & $\begin{array}{c}0.729 \\
(0.027)\end{array}$ & $\begin{array}{c}0.792 \\
(0.039)\end{array}$ & 0.615 & 0.864 & 0.487 & 0.914 \\
\hline RSFc & $\begin{array}{c}0.703 \\
(0.029)\end{array}$ & $\begin{array}{c}0.758 \\
(0.042)\end{array}$ & 0.663 & 0.795 & 0.500 & 0.885 \\
\hline COX & $\begin{array}{c}0.679 \\
(0.035)\end{array}$ & $\begin{array}{c}0.718 \\
(0.048)\end{array}$ & 0.817 & 0.568 & 0.568 & 0.817 \\
\hline $\mathrm{COX}_{\mathrm{c}}$ & $\begin{array}{c}0.636 \\
(0.036)\end{array}$ & $\begin{array}{c}0.712 \\
(0.047)\end{array}$ & 0.808 & 0.545 & 0.545 & 0.808 \\
\hline DeepSurv & $\begin{array}{c}0.712 \\
(0.029)\end{array}$ & $\begin{array}{c}0.759 \\
(0.042)\end{array}$ & 0.731 & 0.705 & 0.525 & 0.854 \\
\hline RF & $\begin{array}{c}0.719 \\
(0.029)\end{array}$ & $\begin{array}{c}0.771 \\
(0.040)\end{array}$ & 0.625 & 0.818 & 0.480 & 0.89 \\
\hline Logistic & $\begin{array}{c}0.697 \\
(0.035)\end{array}$ & $\begin{array}{c}0.740 \\
(0.050)\end{array}$ & 0.760 & 0.682 & 0.545 & 0.849 \\
\hline ANN & $\begin{array}{c}0.640 \\
(0.037)\end{array}$ & $\begin{array}{c}0.660 \\
(0.050)\end{array}$ & 0.750 & 0.568 & 0.490 & 0.804 \\
\hline GBM & $\begin{array}{c}0.717 \\
(0.030)\end{array}$ & $\begin{array}{c}0.767 \\
(0.040)\end{array}$ & 0.683 & 0.750 & 0.500 & 0.866 \\
\hline
\end{tabular}

A

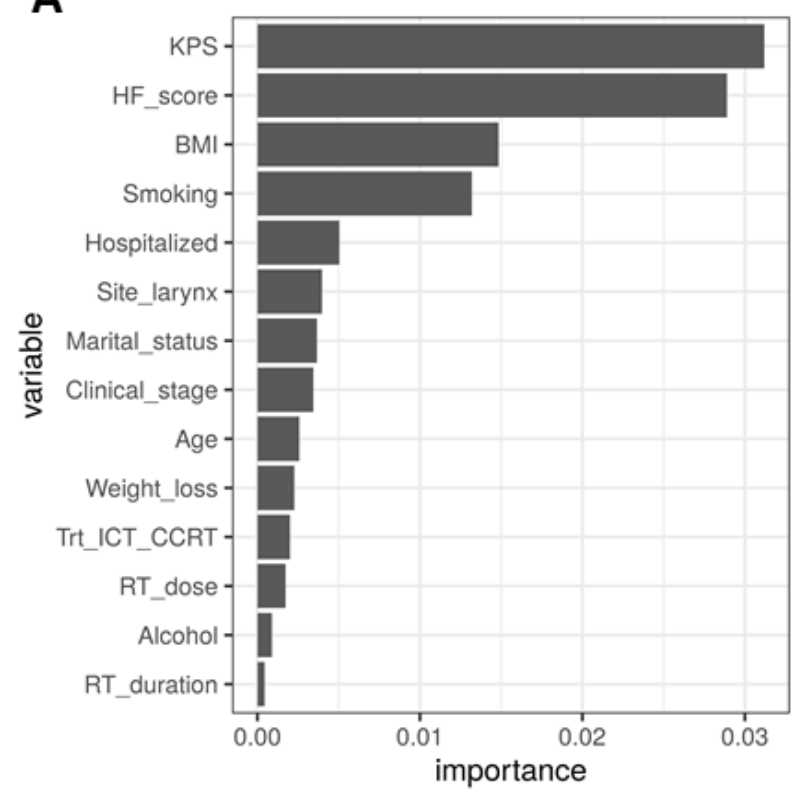

B

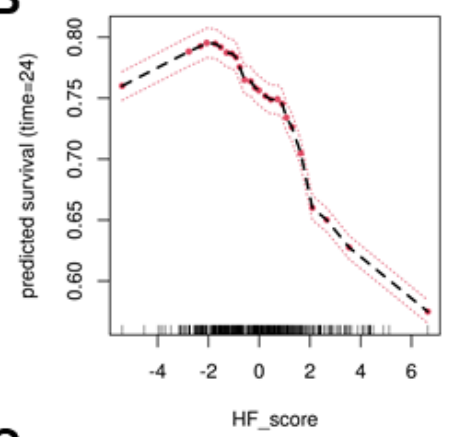

C

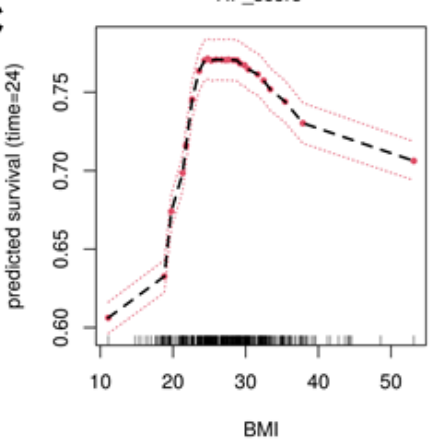

Figure 3. (A) Variable importance for the top predictors of the final model. The HF score is among the top ranked variable. (B) The partial dependence plot of the BMI with the two-year survival rate as outcome. (C) The partial dependence plot of the HF score with the two-year survival rate as outcome.

\subsection{Patient Stratification by HF Score and RSF Predicted Risk}

We further stratified the test cohort patients based on the 40th and 85th percentiles of the predicted mortality by RSF. The cutoff values were previously used by Valero et al. for the H-index they derived for the stratification of oral cavity cancer patients [5]. We used the same set of cutoffs for comparison purpose. Survival analysis showed that the 
group with highest HF scores has significantly higher risk than the low and middle groups in terms of five-year OS (Figure $4 \mathrm{~A}, p<0.0001)$. The hazard ratios (HRs) are $3.76(95 \%$ CI:1.93-7.32) and 1.93 (1.07-3.50), respectively. The HRs are both slightly higher than that of the H-index (3.22 and 1.47) [5]. The HRs are further improved to 7.41 (3.66-14.98) and 2.58 (1.47-4.51) when stratified by the RSF predicted risk, which combined the HF score and clinical/demographic information (Figure 4B). It should be noted the higher HRs does not mean the HF score outperforms the H-index, as the underlying patient population is different. Instead, our results verified the finding that host factors are predictive of head and neck cancer patients' OS.

A

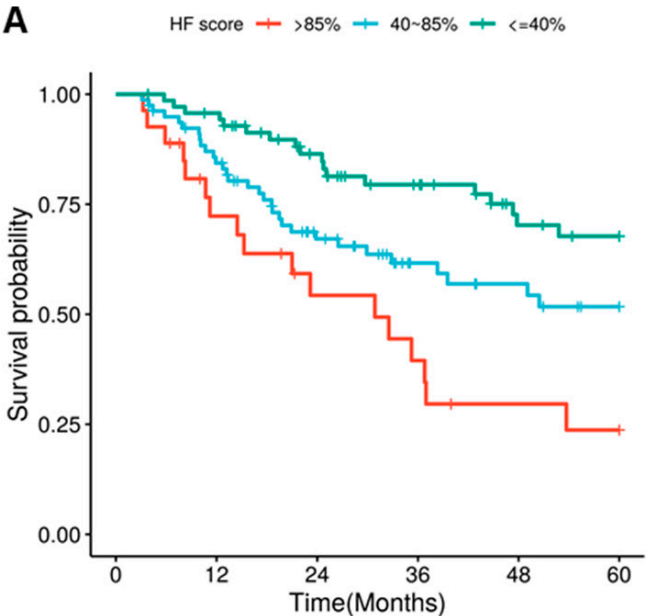

Number at risk

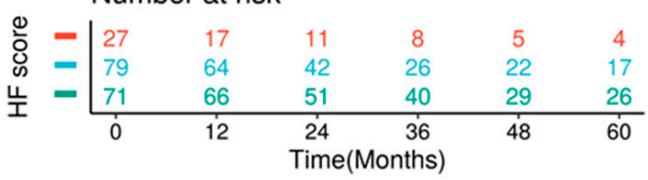

B $\quad$ Risk $+>85 \%+40-85 \%+<=40 \%$

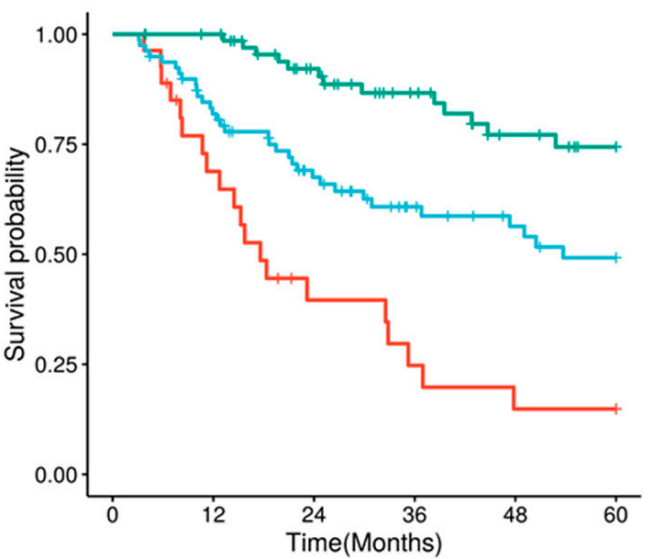

Number at risk

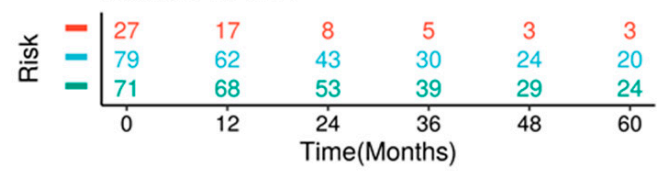

Figure 4. HF score and RSF prediction stratify the test cohort patients into distinct risk groups. The Kaplan-Meier estimates for the patient stratified based on the HF score (A) and risk predicted by RSF model (B) based on cutoffs of the 40th and 85th percentiles. The cutoffs were selected to be comparable with the H-index.

\section{Discussion}

In this study, we used machine learning techniques to build a multivariate model for the prediction of OS in HNSCC patients receiving radiation therapy based on an institutional data set. We further evaluated the contribution of host factors in improving the prediction. The selected model was rigorously validated in test datasets separated before any processing and model training steps to ensure the model's robustness.

The result shows that RSF achieved the best performance among all common modeling methods tested. The model achieved a C-index of 0.729, and with all AUCs above 0.7 for year 1 5. In particular, the AUC for two-year OS is 0.792 , and over 0.8 for one-year OS. Notably, the model only relies on measurements that are routinely collected in clinical settings, so it is highly useful in practice. Our work recapitulated that a model that can handle nonlinear associations and complex interactions works better in predicting head and neck cancer patients' OS. Indeed, nonlinear relationships between predictors and survival rates were observed. The deep learning model is capable of automatic feature extraction, so it may be able to learn the relationship among host factors. However, it did not show advantage in our study, which is possibly due to the moderate sample size available. Another contribution of this work is we verified the previous finding that host factors are independently associated with prognosis of head and neck cancer patients [5], and objectively assessed the improvement in prediction performance by their inclusion into the predictive models. To efficiently utilize the host factor information, we proposed the 
application of PCA. The first component naturally provides a composite score of the states of two groups of factors, which makes interpretation of this score biologically convenient. The HF score alone stratifies the HNSCC patients in the test cohort into distinct risk groups with excellent discriminating capacity. This finding also suggests that dimension reduction is crucial for efficient usage of host factor information by machine learning models, at least when the sample size is moderate.

It should be noted that we included different patient subgroups who underwent surgical treatment and received exclusive chemoradiation therapy. The tree based (RSF, RF, GBM) and neural network machine learning models (DeepSurv and ANN) are expected to account for the disparity in associations between predictors and outcomes in different subgroups. On the other hand, if the associations are shared among subgroups the strategy of combining cohorts is expected to boost the model performance. The C-indices for predicting OS in patients with definitive and post-operative radiotherapies are $0.746(0.028)$ and $0.710(0.081)$, respectively. Therefore, the prediction for non-surgical patients is slightly better, though we cannot accurately evaluate the accuracy in surgical patients due to the small sample size. In addition, some prognostic factors such as pathological grading were not found to be independently predictive in our work. This is possibly due to the fact that most patients had moderately and poor differentiated tumors (Table 1).

Recently, a convolutional neural network (CNN) applied to pre-treatment computed tomography (CT) images achieved an AUC of 0.7 for two-year OS, which was trained on a much smaller data set $(n=194)$ [26]. Cozzi et al. used CT-based radiomics and attained a Cindex of 0.9 in predicting the OS locally advanced head and neck cancer patients. However, there were 40 patients in the validation cohort with only three events [27]. Similarly, a study reported a C-index of 0.781 for cancer-related death for oral cancer patients but there are only 11 events in the test cohort [28]. The model development and selection procedures were not described in detail, making it difficult to evaluate the model's generalizability. In addition, the study used cancer recurrence as a predictor, which contributes roughly 0.06 to the prediction accuracy based on the VIMP reported. However, this is not applicable for primary cancer patients at baseline. In another study, Hung et al. used demographic and clinical data to model oral cancer survival as a continuous variable with all censored observations excluded [29]. The study was conducted on a patient cohort spanning from 1975 to 2016 and the year of diagnosis was found as the most predictive feature. Due to the high heterogeneity in diagnostic years and different metrics of performance, the model is not directly comparable with ours [29]. Moreover, the output of these models cannot be directly converted into survival functions or survival probabilities, so the application is quite limited. In another study, Howard et al. investigated the usage of machine learning in the guidance of the adjuvant treatment of head and neck cancers. While other models had better performance on personalized treatment recommendations, RSF showed best performance in predicting the OS with a C-index of 0.695 [23]. This observation is consistent with our results using clinical and demographic features alone (0.703). Other studies used machine learning models to predict different outcomes such as toxicities [30].

None of the above work investigated the roles of host factors in prognostic modeling. Valero et al. derived the $\mathrm{H}$-index based on host factors for oral cavity cancer patients. While the authors did not assess the index's discriminating ability, based on their categorization, the HR between the highest and lowest risk group (top $14.5 \%$ and bottom $38.6 \%$ ) for OS is 3.22, which is lower than the HF score we derived [5]. Note that our results may not be comparable due to the difference in patient groups. However, our result verified that the host factors are independent predictors of head and neck cancer patients' OS.

The study may have some intrinsic limitations due to its retrospective nature. In addition, the model was only internally validated. We are currently building a cohort for validation to ensure the model's generalizability. Based on the stratification by our predictive model, we will be able to provide supportive care for patients likely to have worst outcome, and design trials specifically on the high-risk patients and test novel treatment management paradigms. As a next step, models predicting adverse events or toxicities 
such as kidney injury from Cisplatin will be investigated. Another important direction is to investigate whether host factor can further improve the prediction performance in combination with radiomics.

\section{Conclusions}

In this work, we evaluated common machine learning models in predicting the head HNSCC patients' overall survival based on clinical, demographic features and host factors. We found the random survival forest had best performance among the models evaluated. We further verified that host factors are independently predictive of HNSCC overall survival and proper dimension reduction is an important step for their incorporation into the machine learning models. The score derived from this process also showed excellent discriminating capacity by itself. The results are of critical importance for improved risk stratification of HNSCC patients and providing targeted supportive care for patients who are likely to have worst outcome.

Supplementary Materials: The following are available online at https:/ /www.mdpi.com/article/10 .3390/cancers13184559/s1, File S1: Detailed infromation of Modeling Strategy.

Author Contributions: Conceptualization, H.Y., M.F., S.J.M., A.K.S.; Data curation, M.F., S.J.M., A.J.I., K.E.W., V.G., R.P.M., M.A.K., M.R.M., J.M.C., W.L.H.J., M.E.P., A.K.S.; Formal analysis, H.Y.; Funding acquisition, A.K.S.; Investigation, H.Y., S.J.M., M.F., A.K.S.; Methodology, H.Y.; Project administration, A.K.S.; Resources, A.K.S.; Supervision, A.K.S.; Visualization, H.Y.; Writing—original draft, H.Y., M.F., S.J.M., A.K.S.; Writing—review and editing, H.Y., S.J.M., M.F., A.J.I., K.E.W., V.G., R.P.M., M.A.K., M.R.M., J.M.C., W.L.H.J., M.E.P., A.K.S. All authors have read and agreed to the published version of the manuscript.

Funding: This work was supported by the National Cancer Institute Cancer Center Support Grant P30CA016056. H.Y. was also supported by the National Cancer Institute IOTN Moonshot Grant U24CA232979.

Institutional Review Board Statement: The study was conducted according to the guidelines of the Declaration of Helsinki and approved by the Institutional Review Board (or Ethics Committee) of Roswell Park Comprehensive Cancer Center (EDR-103707 2/28/2018).

Informed Consent Statement: A waiver of consent was obtained from the Institutional Review Board due to the retrospective nature of the study making consent impractical and contacting patients to obtain consent would pose a greater risk than the waiver.

Data Availability Statement: The data underlying this article cannot be shared publicly for the privacy of individuals that participated in the study. The data are available from the corresponding author upon reasonable request.

Acknowledgments: The authors would like to acknowledge Adam Oberkircher and Kelsey Smith for their tireless efforts to provide excellent care of these patients.

Conflicts of Interest: The authors declare no conflict of interest.

\section{References}

1. Chow, L.Q. Head and neck cancer. N. Engl. J. Med. 2020, 382, 60-72. [CrossRef]

2. Bonner, J.A.; Harari, P.M.; Giralt, J.; Azarnia, N.; Shin, D.M.; Cohen, R.B.; Jones, C.U.; Sur, R.; Raben, D.; Jassem, J. Radiotherapy plus cetuximab for squamous-cell carcinoma of the head and neck. N. Engl. J.Med. 2006, 354, 567-578. [CrossRef] [PubMed]

3. Iocca, O.; Farcomeni, A.; Di Rocco, A.; Di Maio, P.; Golusinski, P.; López, S.P.; Savo, A.; Pellini, R.; Spriano, G. Locally advanced squamous cell carcinoma of the head and neck: A systematic review and Bayesian network meta-analysis of the currently available treatment options. Oral Oncol. 2018, 80, 40-51. [CrossRef] [PubMed]

4. Yan, F.; Li, H.; Kaczmar, J.M.; Sharma, A.K.; Day, T.A.; Neskey, D.M.; Pipkorn, P.; Zenga, J.; Graboyes, E.M. Evaluating Adjuvant Therapy With Chemoradiation vs Radiation Alone for Patients With HPV-Negative N2a Head and Neck Cancer. JAMA Otolaryngol. Head Neck Surg. 2020, 146, 1109-1119. [CrossRef] [PubMed]

5. Valero, C.; Zanoni, D.K.; Pillai, A.; Ganly, I.; Morris, L.G.; Shah, J.P.; Wong, R.J.; Patel, S.G. Host Factors Independently Associated With Prognosis in Patients With Oral Cavity Cancer. JAMA Otolaryngol. Head Neck Surg. 2020, 146, 699-707. [CrossRef]

6. Schafer, U.; Micke, O.; Muller, S.B.; Schuller, P.; Willich, N. Hemoglobin as an independent prognostic factor in the radiotherapy of head and neck tumors. Strahlenther. Onkol. 2003, 179, 527-534. [CrossRef] 
7. McCloskey, S.A.; Jaggernauth, W.; Rigual, N.R.; Hicks, W.L., Jr.; Popat, S.R.; Sullivan, M.; Mashtare, T.L., Jr.; Khan, M.K.; Loree, T.R.; Singh, A.K. Radiation treatment interruptions greater than one week and low hemoglobin levels (12 g/dL) are predictors of local regional failure after definitive concurrent chemotherapy and intensity-modulated radiation therapy for squamous cell carcinoma of the head and neck. Am. J. Clin. Oncol. 2009, 32, 587-591. [CrossRef]

8. Terris, D.J.; Dunphy, E.P. Oxygen tension measurements of head and neck cancers. Arch. Otolaryngol. Head Neck Surg. 1994, 120, 283-287. [CrossRef]

9. Hoff, C.M. Importance of hemoglobin concentration and its modification for the outcome of head and neck cancer patients treated with radiotherapy. Acta. Oncol. 2012, 51, 419-432. [CrossRef] [PubMed]

10. Yu, Y.; Wang, H.; Yan, A.; Wang, H.; Li, X.; Liu, J.; Li, W. Pretreatment neutrophil to lymphocyte ratio in determining the prognosis of head and neck cancer: A meta-analysis. BMC Cancer 2018, 18, 383. [CrossRef] [PubMed]

11. Mascarella, M.A.; Mannard, E.; Silva, S.D.; Zeitouni, A. Neutrophil-to-lymphocyte ratio in head and neck cancer prognosis: A systematic review and meta-analysis. Head Neck 2018, 40, 1091-1100. [CrossRef]

12. Cho, Y.; Kim, J.W.; Yoon, H.I.; Lee, C.G.; Keum, K.C.; Lee, I.J. The Prognostic Significance of Neutrophil-to-Lymphocyte Ratio in Head and Neck Cancer Patients Treated with Radiotherapy. J. Clin. Med. 2018, 7, 512. [CrossRef] [PubMed]

13. Hanahan, D.; Coussens, L.M. Accessories to the crime: Functions of cells recruited to the tumor microenvironment. Cancer Cell 2012, 21, 309-322. [CrossRef]

14. Breiman, L. Random forests. Mach. Learn. 2001, 45, 5-32. [CrossRef]

15. Ishwaran, H.; Kogalur, U.B.; Blackstone, E.H.; Lauer, M.S. Random survival forests. Ann. Appl. Stat. 2008, 2, 841-860. [CrossRef]

16. Katzman, J.L.; Shaham, U.; Cloninger, A.; Bates, J.; Jiang, T.; Kluger, Y. DeepSurv: Personalized treatment recommender system using a Cox proportional hazards deep neural network. BMC Med. Res. Methodol. 2018, 18, 24. [CrossRef] [PubMed]

17. Tibshirani, R. Regression shrinkage and selection via the lasso. J. R. Stat. Soc. Ser. B Stat. Methodol. 1996, 58, 267-288. [CrossRef]

18. Chen, T.; He, T.; Benesty, M.; Khotilovich, V.; Tang, Y.; Cho, H.; Chen, K.; Mithcell, R.; Cano, I.; Zhou, T.; et al. xgboost: Extreme Gradient Boosting. R package version 1.4.1.1. Available online: https:// cran.r-project.org/package=xgboost (accessed on 1 May 2021).

19. Günther, F.; Fritsch, S. Neuralnet: Training of neural networks. R J. 2010, 2, 30-38. [CrossRef]

20. Ishwaran, H.; Kogalur, U.B. randomForestSRC: Fast Unified Random Forests for Survival, Regression and Classification (RF-SRC). R package version 2.12.0. Available online: https:/ / cran.r-project.org/package=randomForestSRC (accessed on 9 July 2021).

21. Hastie, T.; Qian, J. glmnet: Lasso and Elastic-Net Regularized Generalized Linear Models. R package version 4.1-2. Available online: https: / / cran.r-project.org/package=glmnet (accessed on 27 June 2021).

22. Fotso, S. PySurvival: Open source package for survival analysis modeling. 2019. Available online: https://www.pysurvival.io/ (accessed on 1 June 2021).

23. Howard, F.M.; Kochanny, S.; Koshy, M.; Spiotto, M.; Pearson, A.T. Machine Learning-Guided Adjuvant Treatment of Head and Neck Cancer. JAMA Netw. Open 2020, 3, e2025881. [CrossRef]

24. Bice, N.; Kirby, N.; Bahr, T.; Rasmussen, K.; Saenz, D.; Wagner, T.; Papanikolaou, N.; Fakhreddine, M. Deep learning-based survival analysis for brain metastasis patients with the national cancer database. J. Appl. Clin. Med. Phys. 2020, 21, 187-192. [CrossRef]

25. Chen, J.-B.; Yang, H.-S.; Moi, S.-H.; Chuang, L.-Y.; Yang, C.-H. Identification of mortality-risk-related missense variant for renal clear cell carcinoma using deep learning. Ther. Adv. Chronic Dis. 2021, 12, 2040622321992624. [CrossRef]

26. Diamant, A.; Chatterjee, A.; Vallières, M.; Shenouda, G.; Seuntjens, J. Deep learning in head \& neck cancer outcome prediction. Sci. Rep. 2019, 9, 1-10.

27. Cozzi, L.; Franzese, C.; Fogliata, A.; Franceschini, D.; Navarria, P.; Tomatis, S.; Scorsetti, M. Predicting survival and local control after radiochemotherapy in locally advanced head and neck cancer by means of computed tomography based radiomics. Strahlenther. Onkol. 2019, 195, 805-818. [CrossRef] [PubMed]

28. Kim, D.W.; Lee, S.; Kwon, S.; Nam, W.; Cha, I.-H.; Kim, H.J. Deep learning-based survival prediction of oral cancer patients. Sci. Rep. 2019, 9, 1-10. [CrossRef] [PubMed]

29. Hung, M.; Park, J.; Hon, E.S.; Bounsanga, J.; Moazzami, S.; Ruiz-Negrón, B.; Wang, D. Artificial intelligence in dentistry: Harnessing big data to predict oral cancer survival. World J. Clin. Oncol. 2020, 11, 918-934. [CrossRef] [PubMed]

30. Reddy, J.; Lindsay, W.; Berlind, C.; Ahern, C.; Holmes, A.; Smith, B.; Phan, J.; Frank, S.; Gunn, G.; Rosenthal, D. Applying a machine learning approach to predict acute radiation toxicities for head and neck cancer patients. Int. J. Radiat. Oncol. Biol. Phys. 2019, 105, S69. [CrossRef] 